\title{
The University of Wisconsin Space Science and Engineering Center Absolute Radiance Interferometer (ARI): Instrument Overview and Radiometric Performance
}

Joe K. Taylor ${ }^{1}$, Henry E. Revercomb ${ }^{1}$, Henry Buijs ${ }^{2}$, Frederic J Grandmont ${ }^{2}$, P. Jonathan Gero ${ }^{1}$, Fred A. Best ${ }^{1}$, David C. Tobin ${ }^{1}$, Robert O. Knuteson ${ }^{1}$

1 Space Science and Engineering Center, University of Wisconsin-Madison,

${ }^{2}$ ABB-Bomem 


\section{Summary}

- The University of Wisconsin-Madison Space Science and Engineering Center (UWSSEC) and Harvard University (HU) submitted a successful joint proposal entitled "A New Class of Advanced Accuracy Satellite Instrumentation (AASI) for the CLARREO Mission" to the NASA Instrument Incubator Program (IIP). The UW-SSEC / HU team has a long history with the scientific and measurement concepts that have formed the foundation for climate benchmark measurements from space

- The objective of this effort is to advance the technological development of advanced accuracy instrumentation for the measurement of absolute spectrally resolved infrared radiances $(5-50 \mu \mathrm{m})$ with high accuracy $(<0.1 \mathrm{~K}, \mathrm{k}=3$, brightness temperature at scene temperature) for climate benchmark measurements from space

- The UW-SSEC, developed a demonstration test bed which includes an FTS instrument and calibration and validation system to demonstrate the feasibility of the far and mid infrared instrumentation for a Climate Benchmark Mission. 


\section{Topics}

1. Introduction

2. The UW-SSEC Absolute Radiance Interferometer (ARI)

3. Radiometric Performance

4. Conclusion 
1. Introduction

2. The UW-SSEC Absolute Radiance Interferometer (ARI)

3. Radiometric Performance

4. Conclusion 


\section{Climate Benchmark Measurements}

- Satellite Instrument Calibration for Measuring Global Climate Change (NIST Publication NISTIR 7047, 2003)

- $\mathrm{ASIC}^{3}$ Report: Achieving Satellite Instrument Calibration for Climate Change (2007)

- US NRC Decadal Survey (NRCDS, 2007): Earth science and applications from space: national imperatives for the next decade and beyond

- Climate Absolute Radiance and Refractivity Observatory (CLARREO): Tier 1 (highest priority) mission

- NASA Implementation of CLARREO

- Selected for development/implementation by NASA (lead: NASA LaRC)

- Successful MCR (November 2010)

- Guidance received in the President's FY 2012 budget removed \$1.24B from the $\$ 2.08 \mathrm{~B} F \mathrm{FY}^{\prime} 11$ proposed Climate Initiative ... directed cuts include CLARREO

- Zeus 


\section{Capability of Current Systems}

- Current generation of high resolution IR sounders: AIRS, IASI, CrIS...

- Tremendous advance in information content \& accuracy

- Huge advance for climate process studies

- Provide a solid foundation for IR Climate Benchmark Measurement feasibility

- But, not optimized for unequivocal decadal trending of climate change, and for the most part, are not designed to provide:

- The radiometric accuracy and sampling required to detect the small trends associated with global climate change

- On-orbit calibration traceability to absolute standards

- Far infrared (FIR) coverage beyond the normal IR sounding region (typically some part or all of the 3-15 $\mu \mathrm{m}$ region) 


\section{Requirements for IR Climate Benchmark Measurements}

- Absolute Accuracy: $<0.1 \mathrm{~K}, \mathrm{k}=2$, brightness temperature for combined measurement and sampling uncertainty for annual averages of $15^{\circ}$ zones to approach goal of resolving a climate change signal in the decadal time frame

- Measurement uncertainty: $<0.1 \mathrm{~K}, \mathrm{k}=3$

- Sampling uncertainty: $<0.1 \mathrm{~K}, \mathrm{k}=3$

- On-orbit Verification and Test: Provide an On-orbit Absolute Radiance/Brightness Temperature Standard with an accuracy of $<0.1 \mathrm{~K}, \mathrm{k}=3$, to provide SI traceability of on-orbit measurements

- Spectral Coverage and Resolution: $200-2000$ (goal: 3000$) \mathrm{cm}^{-1} ; \sim 0.5 \mathrm{~cm}^{-1}( \pm 1$ $\mathrm{cm}$ MOPD)

- Spatial Footprint \& Angular Sampling: Order 100 km or less, nadir only

- Coverage: Contiguous coverage not required 
1. Introduction

2. The UW-SSEC Absolute Radiance Interferometer (ARI)

3. Radiometric Performance

4. Conclusion 


\section{Technology Developments Under NASA IIP}

Calibration Reference: Ambient Blackbody (ABB)

Used in conjunction with $H B B$ for instrument calibration

On-orbit Cavity Emissivity Module (OCEM)

- Heated Halo

- $Q C L$
Absolute Radiance Interferometer (ARI)

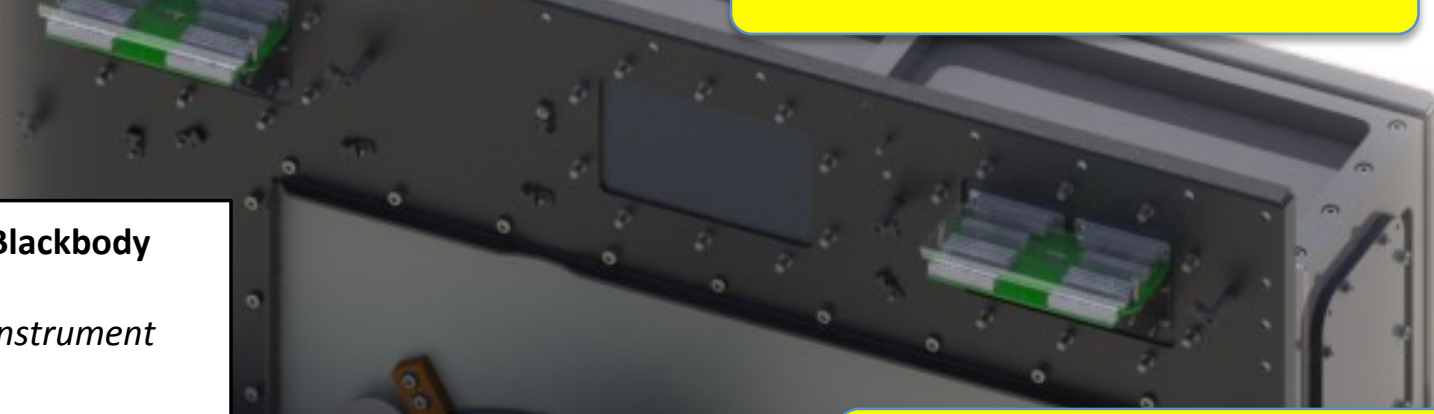

On-orbit Spectral Response Module (OSRM)

- Instrument Lineshape measurement

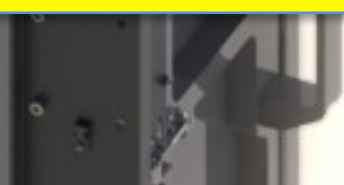

Calibration Reference: Hot Blackbody (HBB)

Used in conjunction with $A B B$ for instrument

calibration

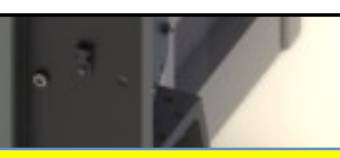

On-orbit Absolute Radiance Standard (OARS)

- Includes Multiple Miniature Phase Change Cells for absolute temperature calibration and Heated Halo OCEM for spectral reflectance measurement

Fred Best: "On-Orbit Absolute Radiance Standard for the Next Generation of IR Remote Sensing Instruments (Monday, 13:35 - 13:55) Jon Gero: "The Heated Halo for Space-Based Blackbody Emissivity Measurement" (Tuesday, 16:30 - 16:50) 


\section{UW-SSEC Spectrometer, Blackbody Heritage \& Ties to NIST}

Ground-based

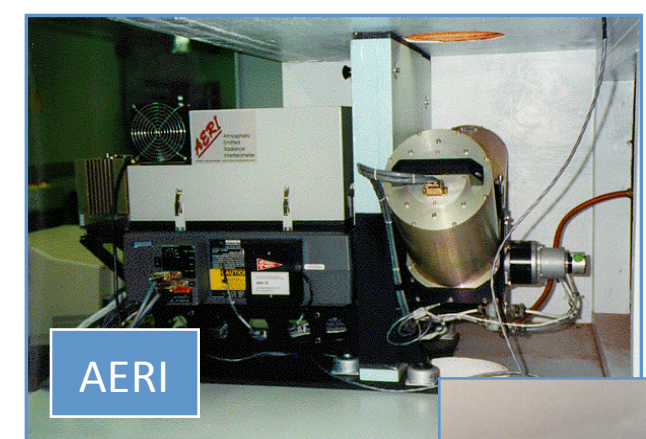

NIST

Water-bath

Blackbody
High-altitude Aircraft
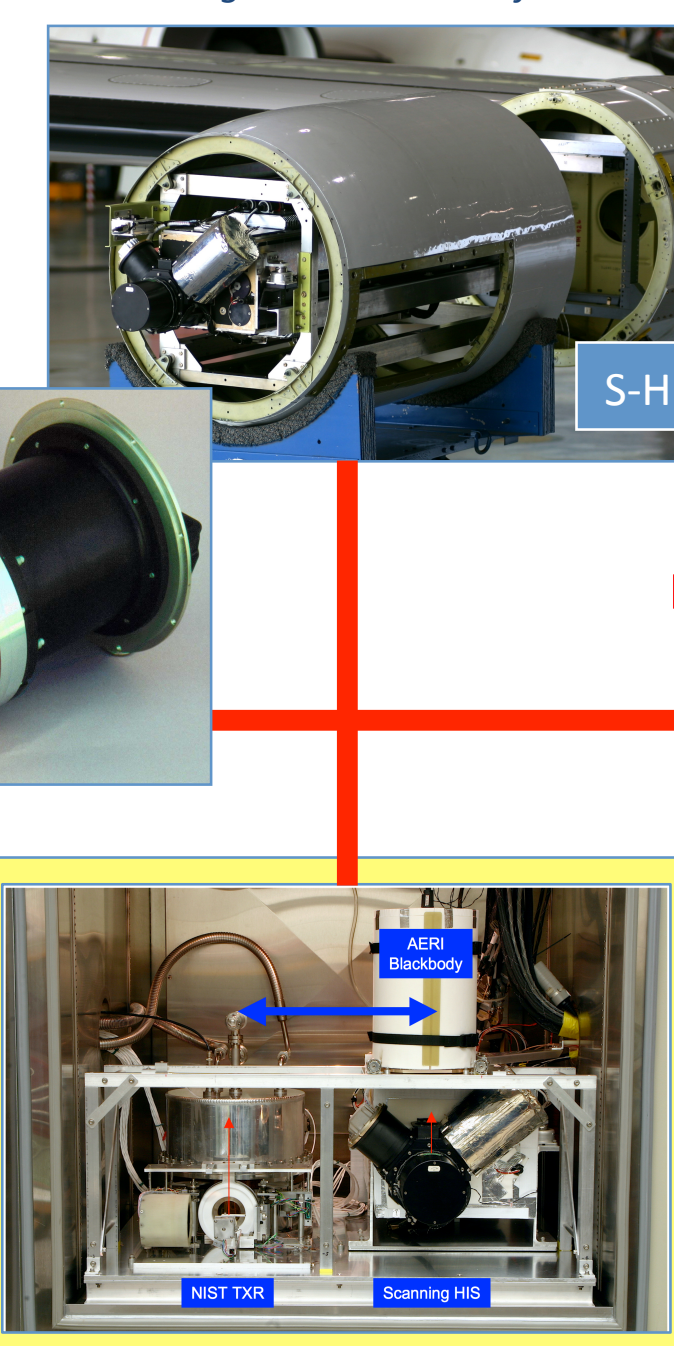

$<0.06$ K error (220 to 333 K)
Spaceflight
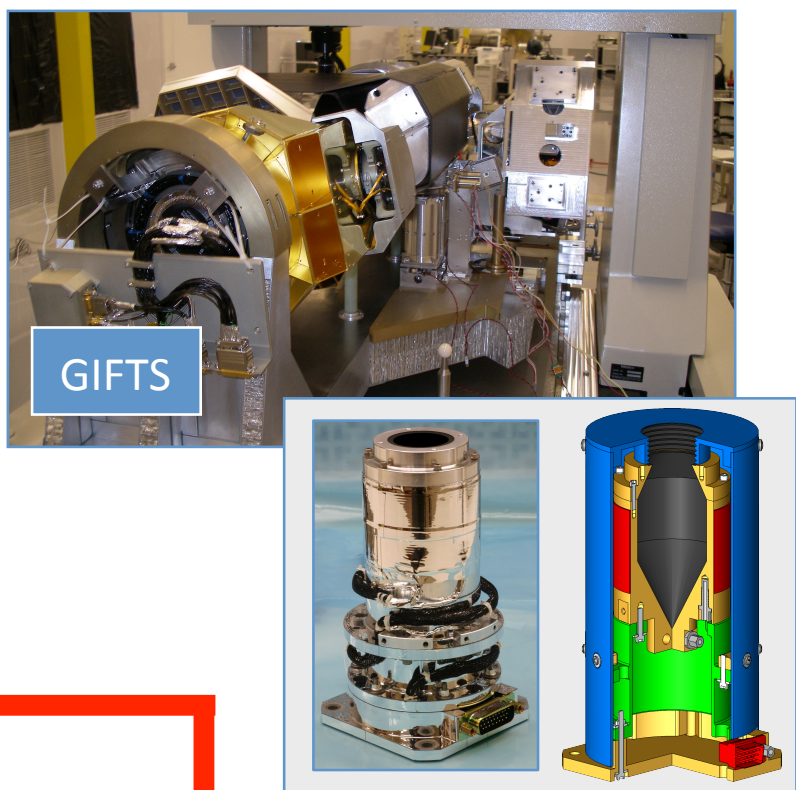

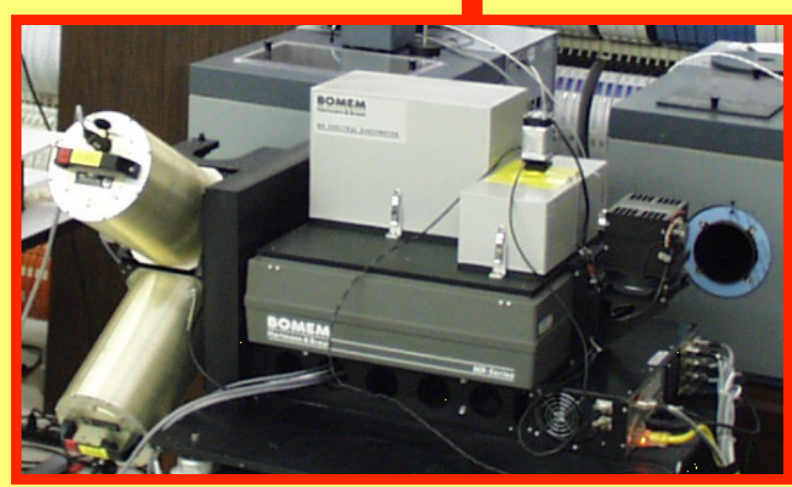

$<0.065$ K error (293 to 333 K)

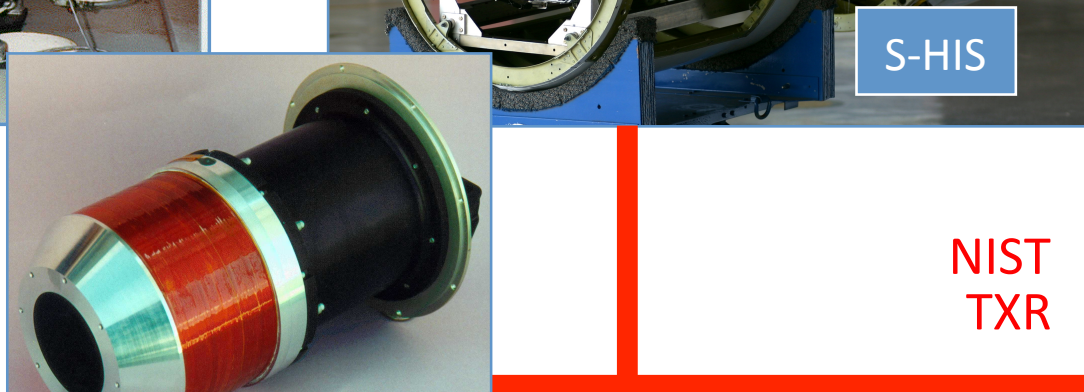

TXR

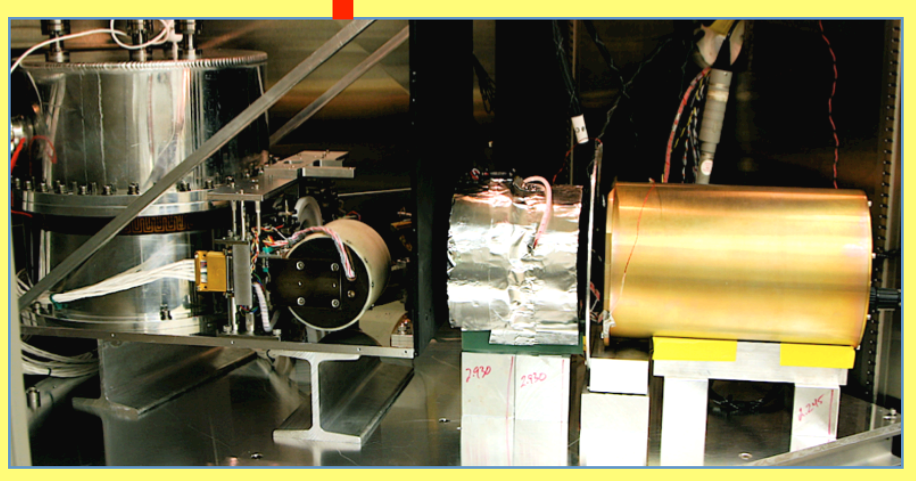

$\varepsilon>0.9994$ (within estimated uncertainty) 


\section{UW-SSEC Absolute Radiance Interferometer}

- The UW-SSEC Absolute Radiance Interferometer includes:

- A scene selection mirror assembly;

- Fore optics designed specifically for high radiometric accuracy;

- A 4-port cube corner, rocking arm interferometer with a diode laser based metrology system;

- Two aft optics assemblies, 1 at each output port of the interferometer;

- A $77 \mathrm{~K}$ multiple semi-conductor detector $\left(400-3000 \mathrm{~cm}^{-1}\right)$ and dewar assembly, and associated mechanical cooler;

- A DTGS pyroelectric detector $\left(200-1800 \mathrm{~cm}^{-1}\right)$ assembly.

Each chosen for their strong spaceflight heritage such that detailed performance testing can be conducted on a system with a clear path to space. For compatibility with an IIP budgets, the electronics are not flight designs 


\section{The Generic Flight Interferometer (GFI)}

- The UW ARI is based on ABB's Generic Flight Interferometer (GFI) architecture: a flex blade-based frictionless double pendulum scanning mechanism with 25 years of heritage and a direct evolution of 2 successful spaceborne interferometers:

- SCISAT / ACE-FTS (2003): Initial design life of 2 years and still operating in compliance with performance requirements after 9 years

- GOSAT / TANSO-FTS (2009): Currently meets all performance requirements in flight

- Additionally, the GFI baseline includes:

- Fiber-linked metrology for reduced heat load on interferometer and simplified alignment / redundancy management

- Monolithic cube corner mirror for increased robustness to launch vibration

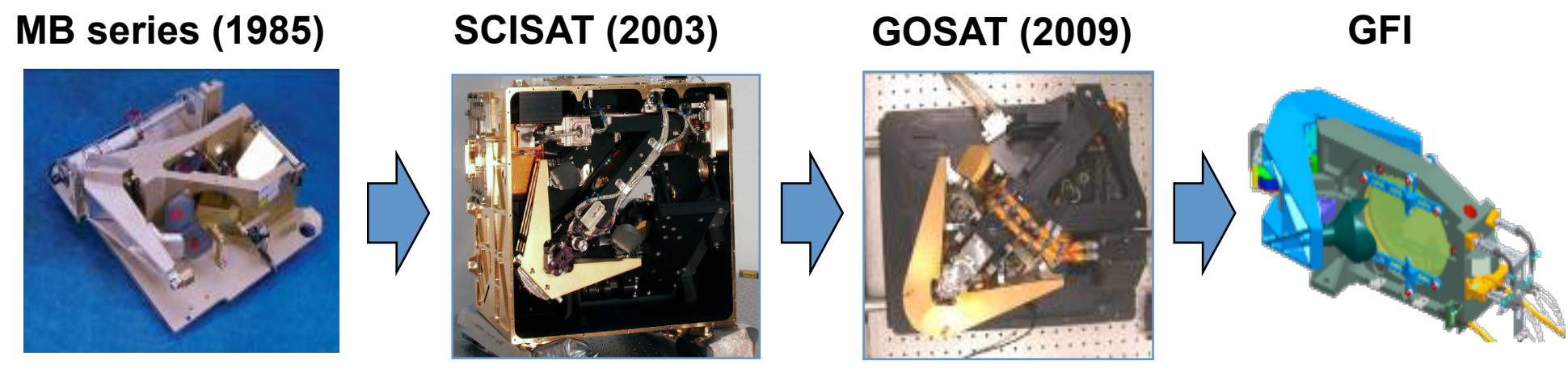




\section{The Generic Interferometer for Climate Studies (GICS)}

- 4 port

- Different met laser path

- Csl beamsplitter to cover spectral range

- Mounting adapted for Csl

- Self compensated beamsplitter instead of substrate and compensator

- Replicated monolithic cube corner

- Vacuum compatible Interferometer

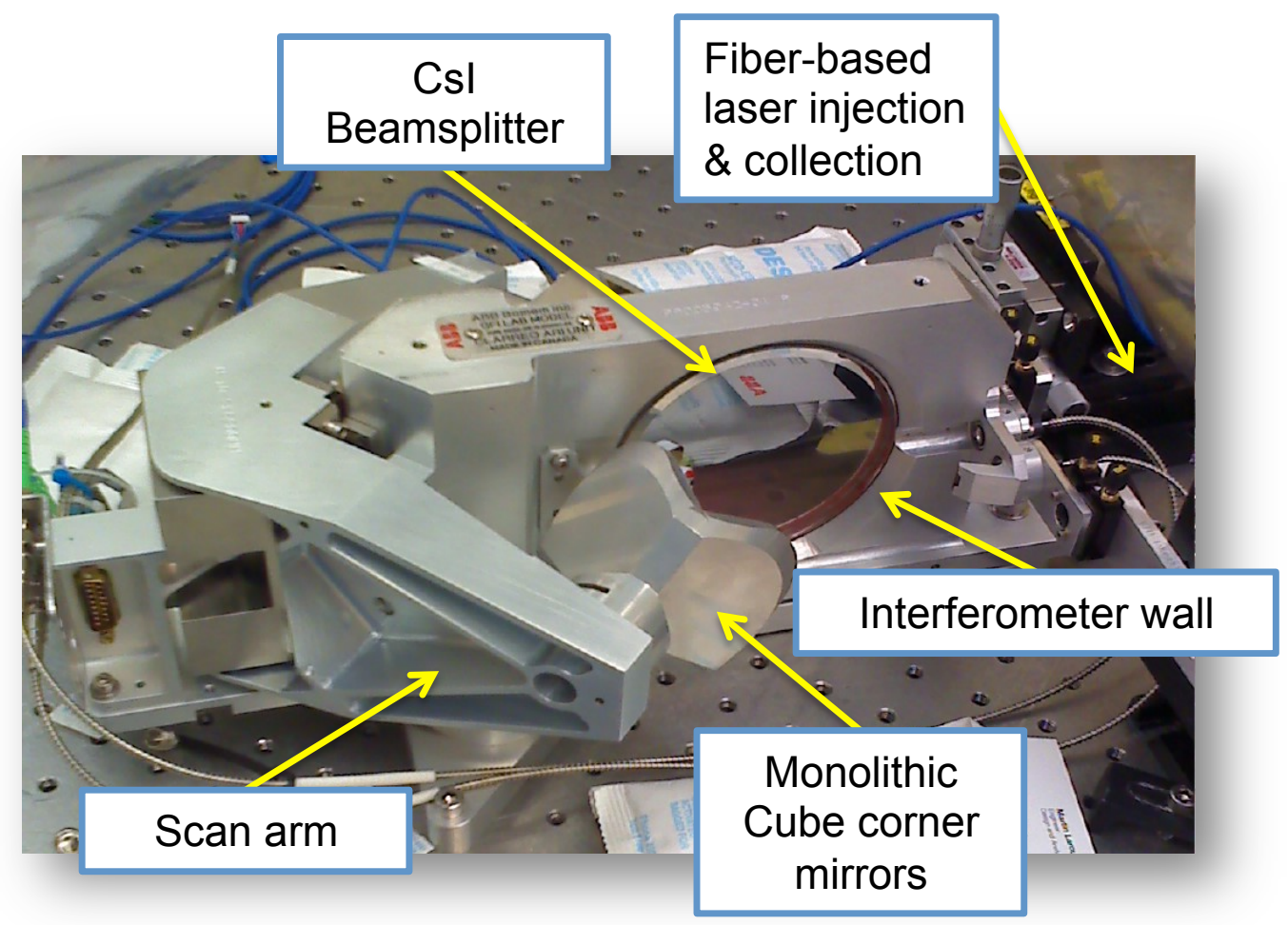

- Modified COTS electronics and software used for IIP

- Mass: < 7 kg (GICS, Aluminum)

- Power: Avg 18 W / Pk 23 W (flight design) 


\section{Optical Design}

Gold coated Aluminum (no AR coating) reflective components

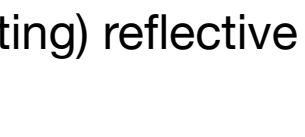

Design goals included:

- Optimize interferometer throughput

- Maximize Stray light control

- Minimize instrument mass and volume

- Optimize heated halo fill factor, $f$

Scene Mirror

- Compatible with 1" aperture Blackbody

- $\quad$ Allow 'tuning' of polarization null locations

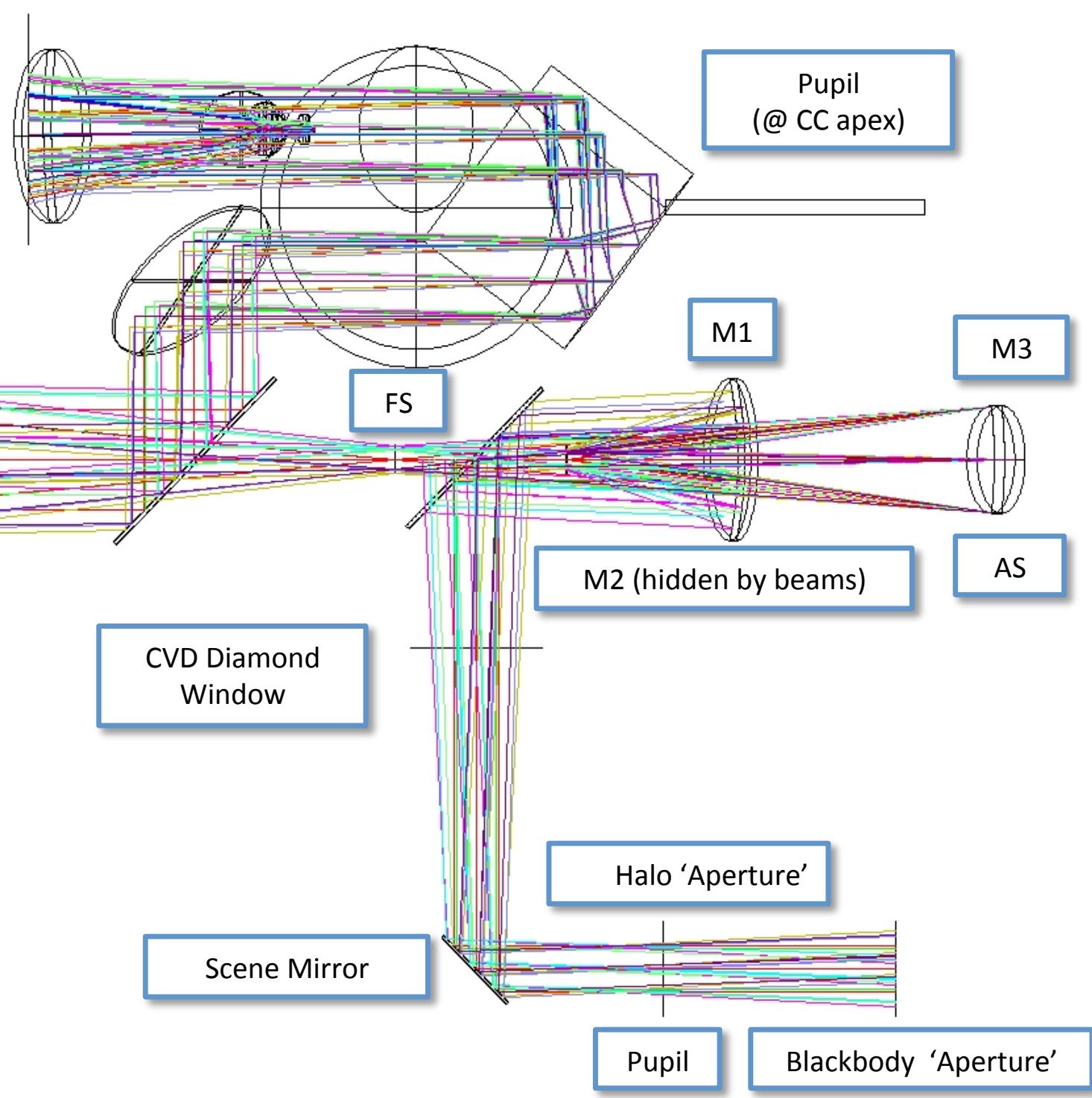




\section{Absolute Radiance Interferometer}

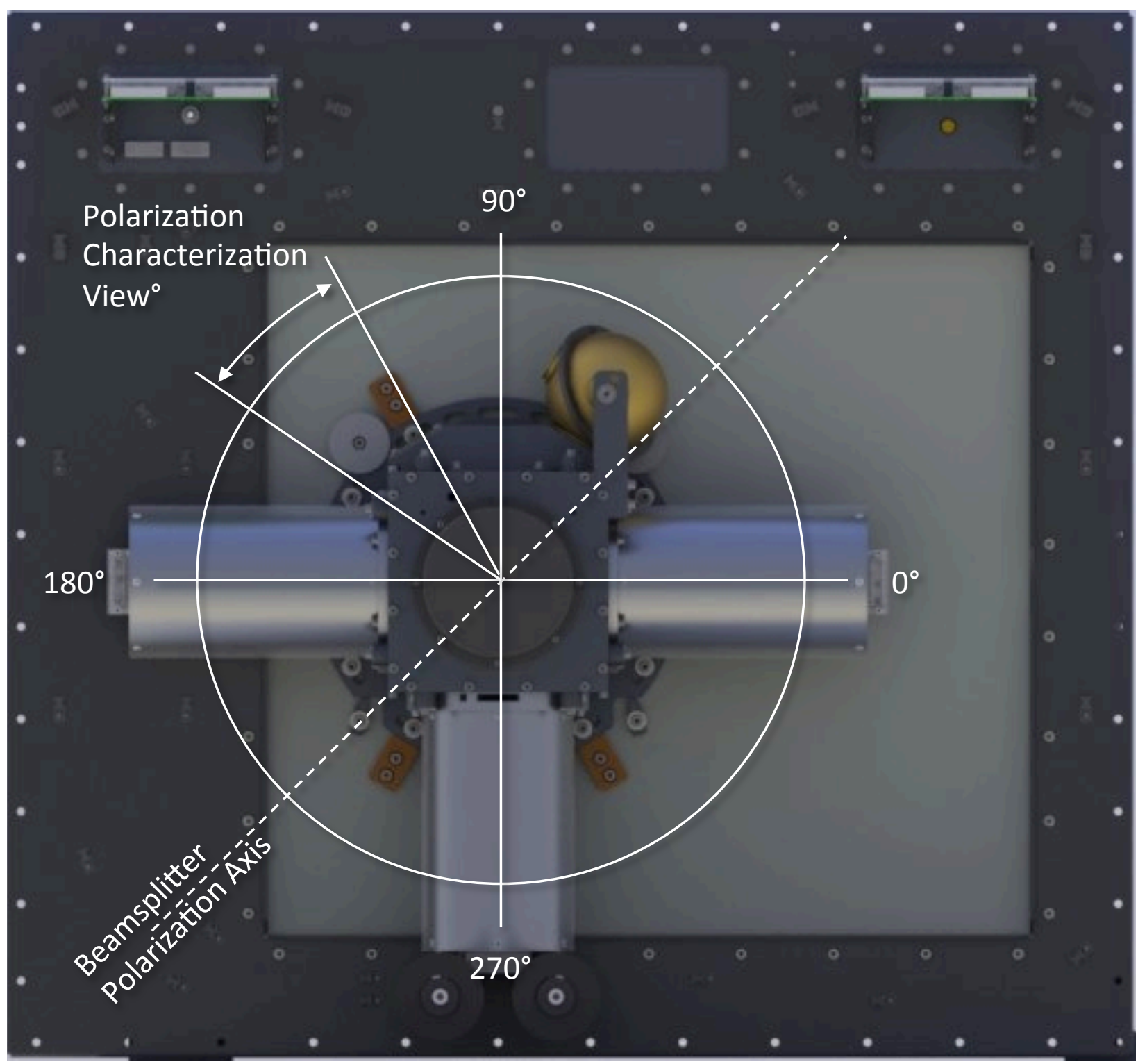

Viewing configuration providing immunity to polarization effects. 


\section{Absolute Radiance Interferometer}

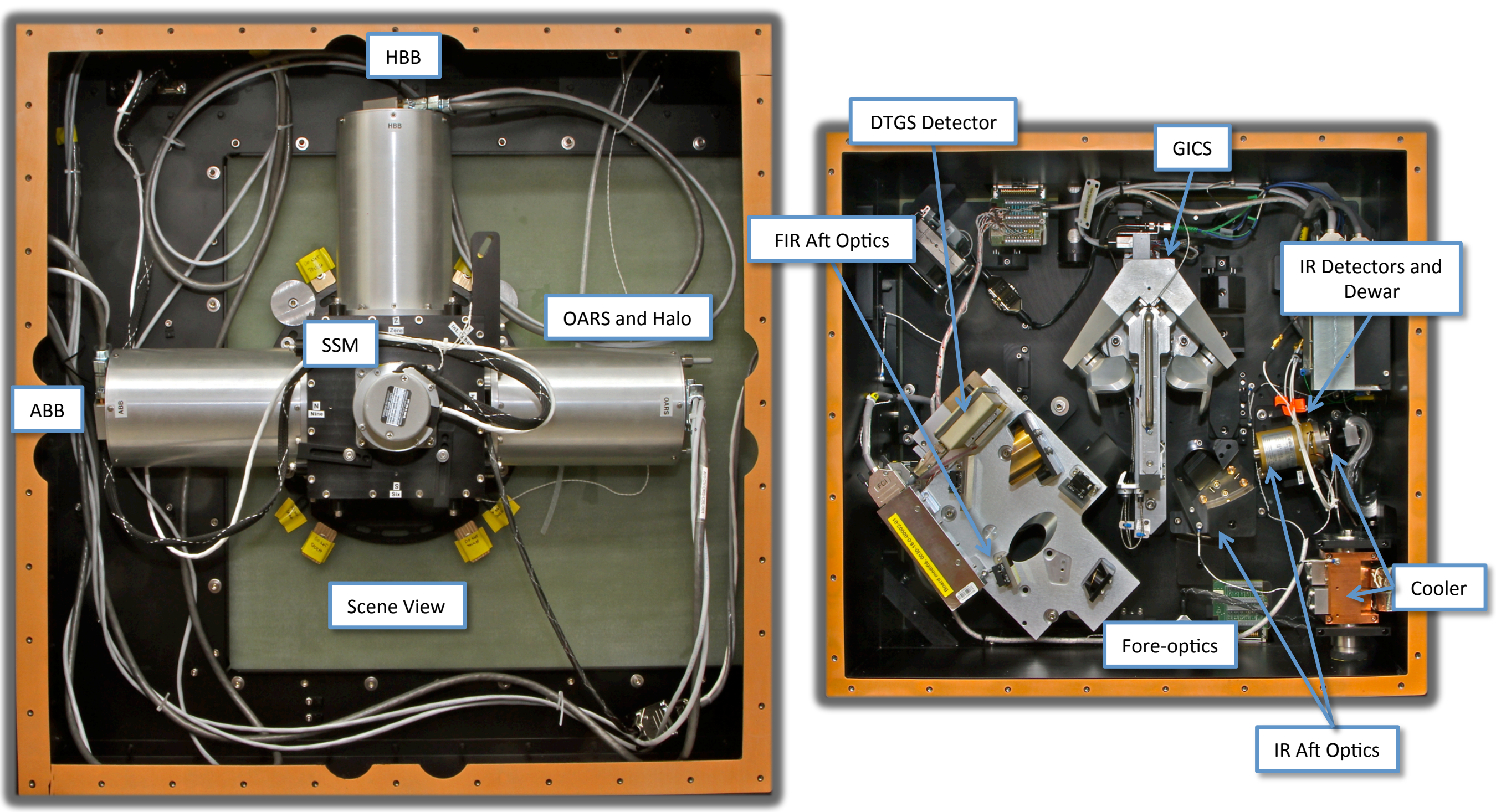

Completed Prototype 
1. Introduction

2. The UW-SSEC Absolute Radiance Interferometer (ARI)

3. Radiometric Performance

4. Conclusion 


\section{Instrument Testing}

- Near Field Response Mapping

- Detector Performance Testing and Demonstrations

- Interferometric Noise Testing and Analysis

- Spectral Calibration Verification

- Nonlinearity Characterization and Analysis

- Radiometric Calibration Verification: OARS

- Radiometric Calibration Verification: Ice Bath Blackbody

- OCEM Heated Halo 


\section{Radiometric Uncertainty}

- Recall the basic calibration expression for the complex calibration method:

$$
\begin{gathered}
N=\left(L_{H}-L_{C}\right) \operatorname{Re}\left\{\frac{C_{S}-C_{C}}{C_{H}-C_{C}}\right\}+L_{C}, \\
L=e B(T)+(1-e) B\left(T_{R}\right)
\end{gathered}
$$

- Radiometric uncertainty estimate: Differential error analysis (and/or perturbation analysis) of the calibration equation

- The uncertainty in the verification of the calibrated radiance includes a contribution from the uncertainty in the determination of the predicted radiance in addition to the uncertainty in the measured radiance 


\section{Radiometric Uncertainty (Predicted On-orbit)}

- On-orbit:

- Space view for cold calibration reference

- Onboard ambient calibration blackbody for "hot" calibration reference

- These values satisfy the Zeus/CLARREO accuracy requirements

\begin{tabular}{|c|c|c|c|c|c|}
\hline \multicolumn{3}{|l|}{ Temperatures } & \multicolumn{2}{|c|}{$\begin{array}{l}\text { Associated Uncertainty } \\
(3-\sigma)\end{array}$} & \\
\hline Cold Cal Ref (Space Target) & $T_{C}$ & $4 \mathrm{~K}$ & $u\left(T_{C}\right)$ & $0 \mathrm{~K}$ & \\
\hline Hot Cal Ref (Internal Cal Target) & $T_{H}$ & $295 \mathrm{~K}$ & $u\left(T_{H}\right)$ & $0.045 \mathrm{~K}$ & \multirow{2}{*}{$\begin{array}{c}\text { Cal BB / OARS } \\
\text { Uncertainty Analysis }\end{array}$} \\
\hline Verification Target (OARS) & $T_{\text {OARS }}$ & $220-320 \mathrm{~K}$ & $u\left(T_{\text {OARS }}\right)$ & $0.045 \mathrm{~K}$ & \\
\hline Reflected Radiance, Cold Cal Ref & $T_{R, C}$ & $290 \mathrm{~K}$ & $u\left(T_{R, C}\right)$ & $0 \mathrm{~K}$ & \multirow{7}{*}{$\begin{array}{c}\text { Halo Uncertainty } \\
\text { Analysis }\end{array}$} \\
\hline Reflected Radiance, Hot Cal Ref & $T_{R, H}$ & $290 \mathrm{~K}$ & $u\left(T_{R, H}\right)$ & $4 \mathrm{~K}$ & \\
\hline Reflected Radiance, Verification Target & $T_{R, O A R S}$ & $290 \mathrm{~K}$ & $u\left(T_{R, O A R S}\right)$ & $4 \mathrm{~K}$ & \\
\hline \multicolumn{5}{|l|}{ Emissivities } & \\
\hline Cold Cal Ref (Space Target) & $e_{C}$ & 1 & $u\left(e_{C}\right)$ & 0.0006 & \\
\hline Hot Cal Ref (Internal Cal Target) & $e_{H}$ & 0.999 & $u\left(e_{H}\right)$ & 0.0006 & \\
\hline Verification Target (OARS) & $e_{\text {OARS }}$ & 0.999 & $u\left(e_{\text {OARS }}\right)$ & $0.0006^{*}$ & \\
\hline
\end{tabular}

* For the On-orbit uncertainty analysis, it has been assumed that the OARS emissivity and associated uncertainty is determined from prelaunch TVAC testing with a very high emissivity source

- $e_{\text {OARS }}=0.9990 \pm 0.0006\left(200 \mathrm{~cm}^{-1}\right)$

- $e_{\text {OARS }}=0.9990 \pm 0.0004\left(800 \mathrm{~cm}^{-1}\right)$

- $e_{\text {OARS }}=0.9990 \pm 0.0002\left(1400 \mathrm{~cm}^{-1}\right)$

- $e_{\text {OARS }}=0.9990 \pm 0.0001\left(2000 \mathrm{~cm}^{-1}\right)$

- $e_{\text {OARS }}=0.9990 \pm 0.00075\left(2600 \mathrm{~cm}^{-1}\right)$ 


\section{Radiometric Uncertainty (Predicted On-orbit)}

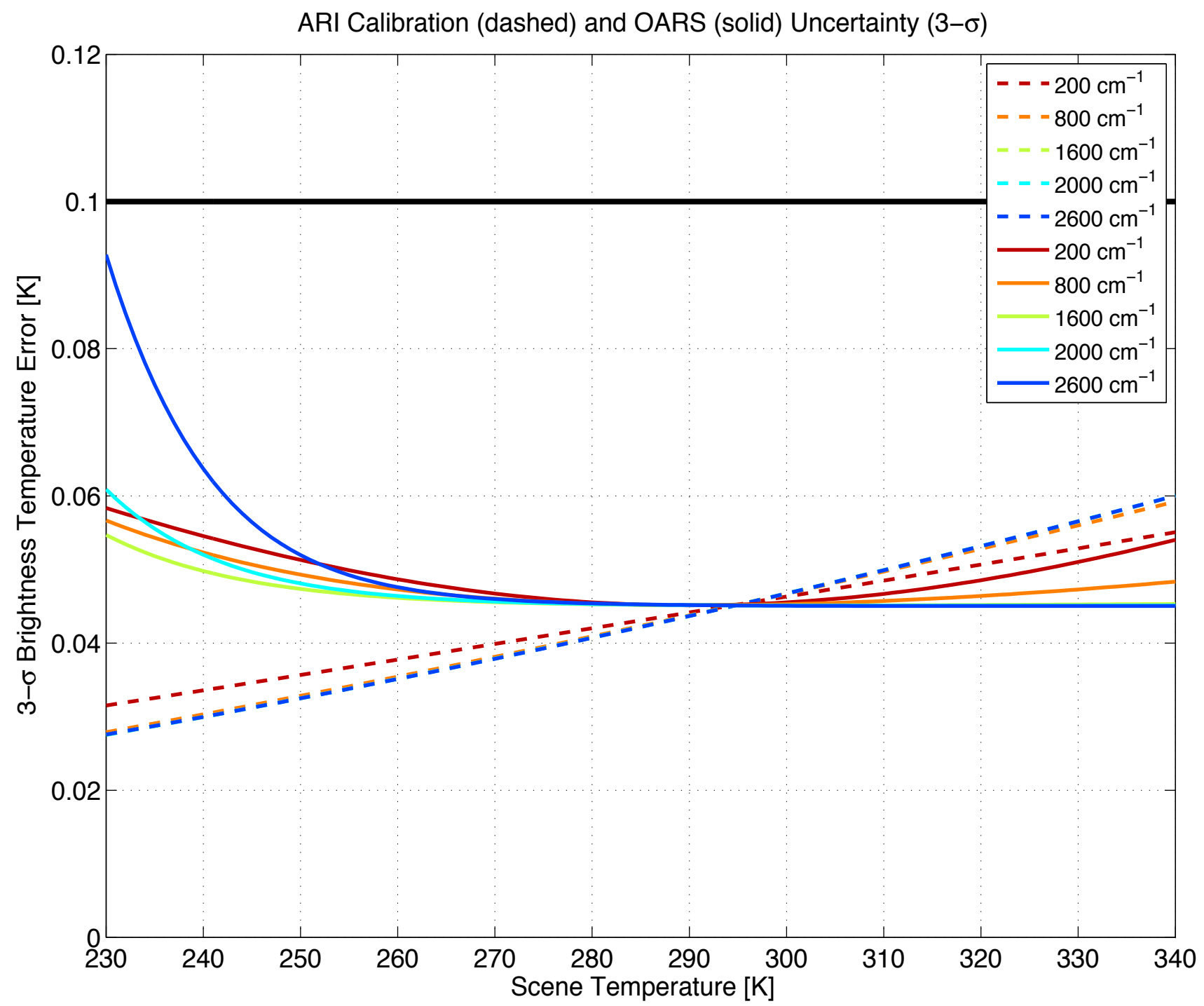

* Uncertainty due to residual nonlinearity not shown 


\section{Combined UW - ARI Calibration and OARS Uncertainty $(k=3)$}

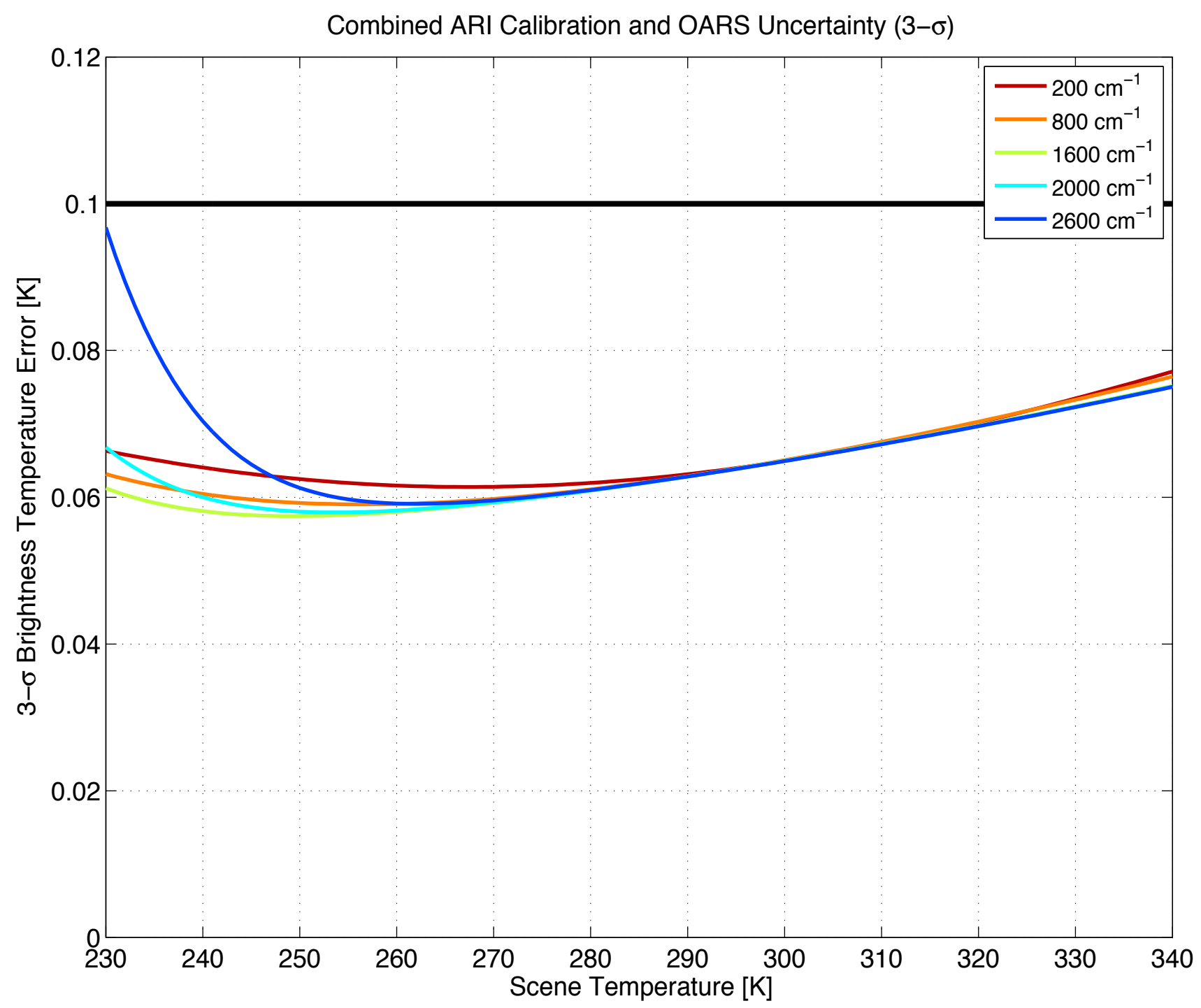

* Uncertainty due to residual nonlinearity not shown 


\section{Radiometric Uncertainty (Laboratory Environment)}

- IIP Demonstration (Laboratory Environment):

- Onboard ambient calibration blackbody for "cold" calibration reference

- Onboard hot calibration blackbody for "hot" calibration reference

\begin{tabular}{|c|c|c|c|c|c|}
\hline \multicolumn{3}{|l|}{ Temperatures } & \multicolumn{2}{|c|}{$\begin{array}{l}\text { Associated Uncertainty } \\
(3-\sigma)\end{array}$} & \\
\hline Cold Cal Ref (Ambient Blackbody) & $T_{C}$ & $293 \mathrm{~K}$ & $u\left(T_{C}\right)$ & $0.045 \mathrm{~K}$ & \\
\hline Hot Cal Ref (Hot Blackbody) & $T_{H}$ & $333 \mathrm{~K}$ & $u\left(T_{H}\right)$ & $0.045 \mathrm{~K}$ & \multirow{2}{*}{$\begin{array}{c}\text { Cal BB / OARS } \\
\text { Uncertainty Analysis }\end{array}$} \\
\hline Verification Target (OARS) & $T_{\text {OARS }}$ & $213-333 \mathrm{~K}$ & $u\left(T_{O A R S}\right)$ & $0.045 \mathrm{~K}$ & \\
\hline Reflected Radiance, Cold Cal Ref & $T_{R, C}$ & $290 \mathrm{~K}$ & $u\left(T_{R, C}\right)$ & $4 \mathrm{~K}$ & \multirow{7}{*}{$\begin{array}{c}\text { Halo Uncertainty } \\
\text { Analysis }\end{array}$} \\
\hline Reflected Radiance, Hot Cal Ref & $T_{R, H}$ & $290 \mathrm{~K}$ & $u\left(T_{R, H}\right)$ & $4 \mathrm{~K}$ & \\
\hline Reflected Radiance, Verification Target & $T_{R, O A R S}$ & $290 \mathrm{~K}$ & $u\left(T_{R, \text { OARS }}\right)$ & $4 \mathrm{~K}$ & \\
\hline \multicolumn{5}{|l|}{ Emissivities } & \\
\hline Cold Cal Ref (Ambient Blackbody) & $e_{C}$ & 0.999 & $u\left(e_{C}\right)$ & 0.0006 & \\
\hline Hot Cal Ref (Hot Blackbody) & $e_{H}$ & 0.999 & $u\left(e_{H}\right)$ & 0.0006 & \\
\hline Verification Target (OARS) & $e_{\text {OARS }}$ & 0.999 & $u\left(e_{O A R S}\right)$ & 0.0006 & \\
\hline
\end{tabular}




\section{Radiometric Uncertainty (Laboratory Environment) UW - ARI Calibration and OARS Uncertainty $(k=3)$}

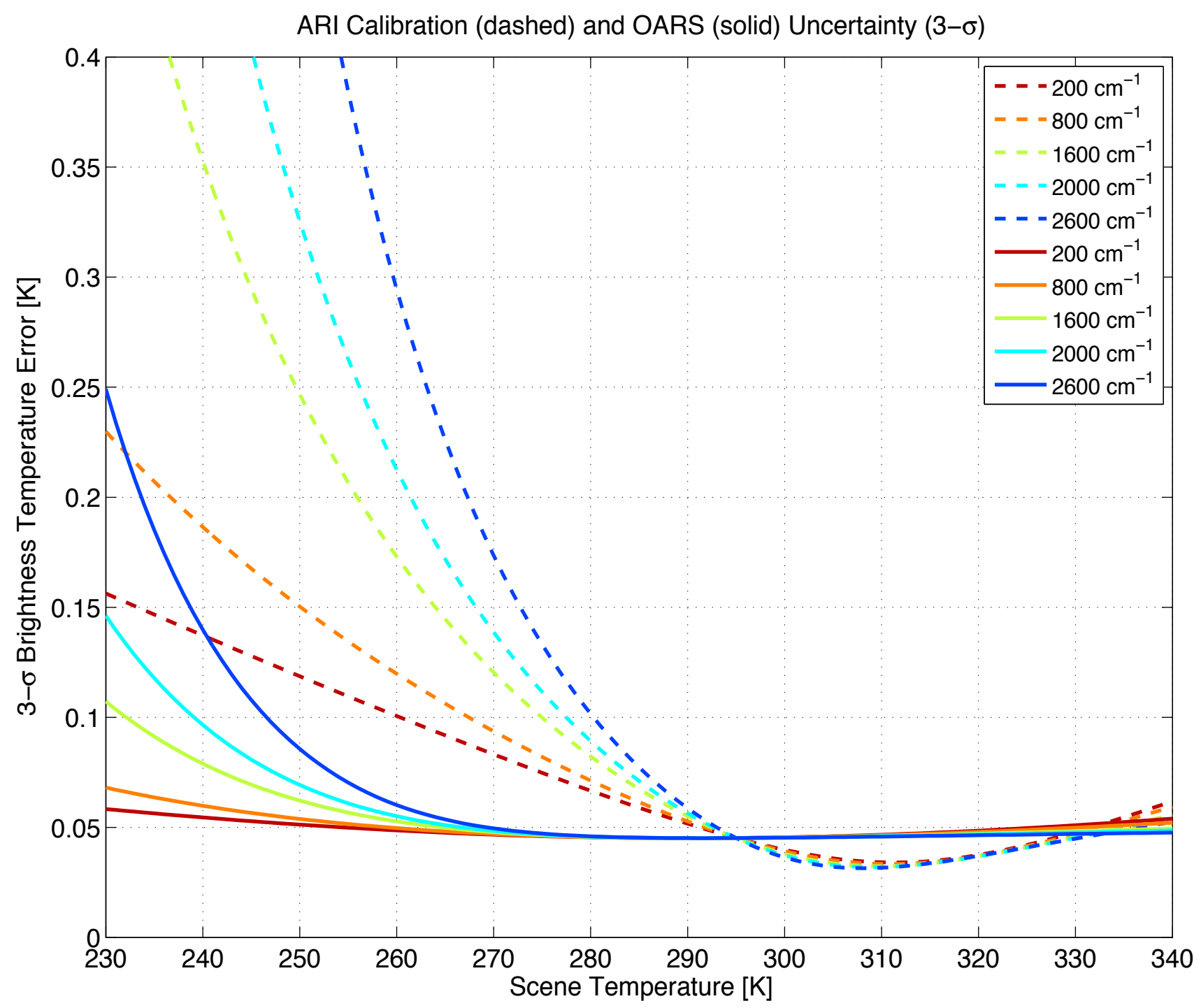


Radiometric Uncertainty (Laboratory Environment)

\section{Combined UW - ARI Calibration and OARS Uncertainty ( $\mathrm{k}=3$ )}

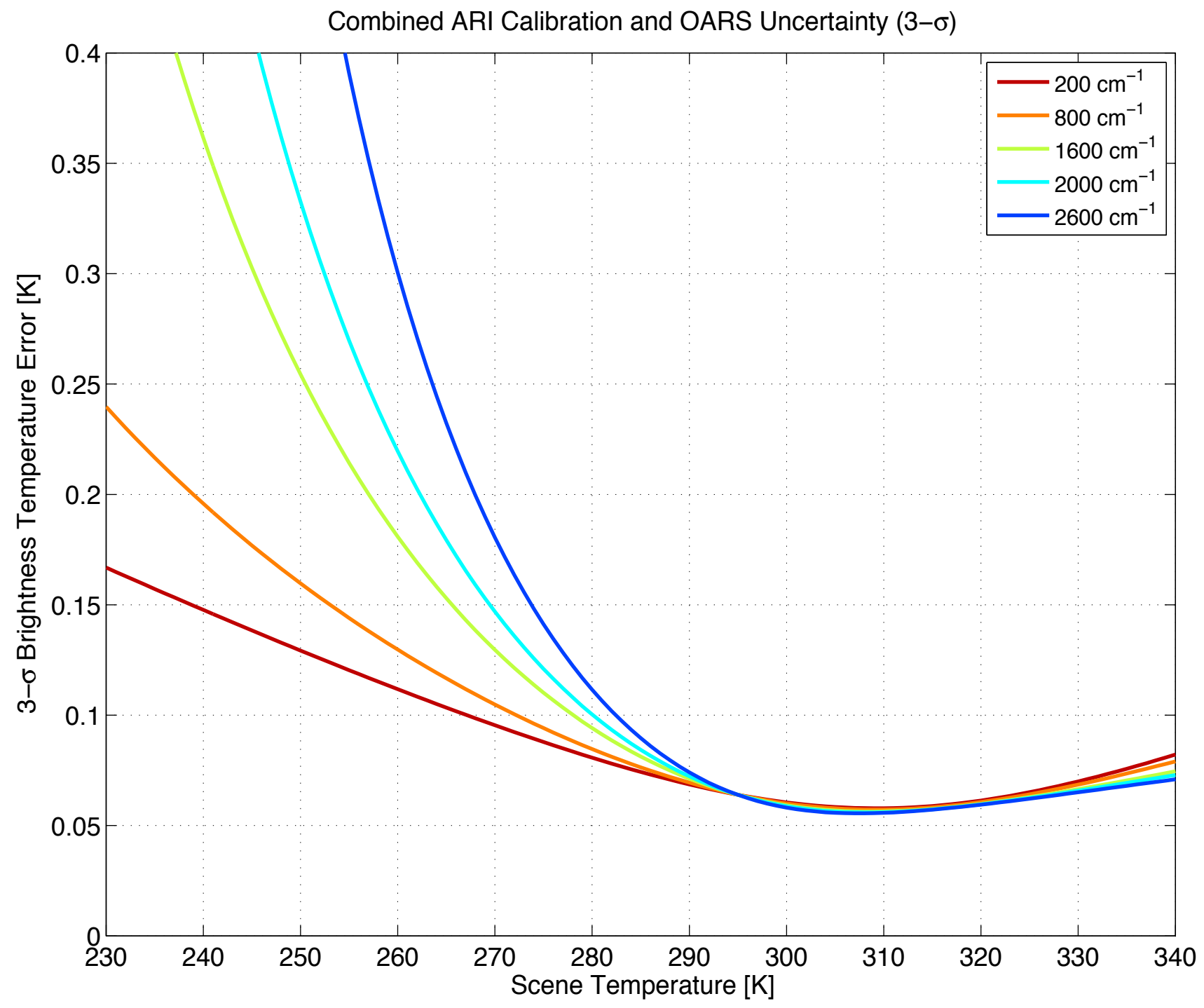

Meeting these uncertainties in the laboratory environment demonstrates the capability to meet the $0.1 \mathrm{~K}(\mathrm{k}=3)$ uncertainty requirement on-orbit 


\section{Radiometric Calibration Verification - MCT with NLC}

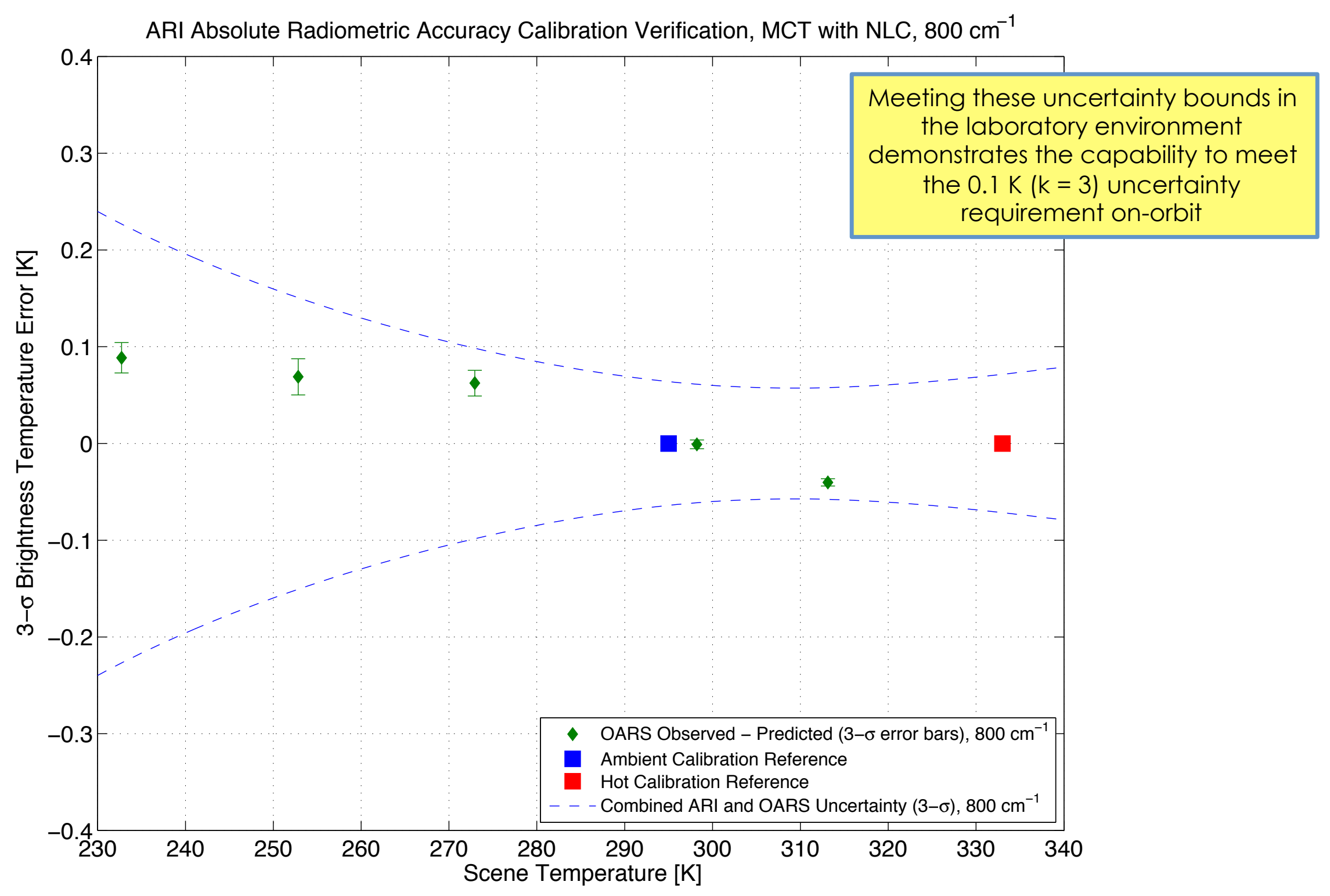




\section{Radiometric Calibration Verification - DTGS $\left(800 \mathrm{~cm}^{-1}\right)$}

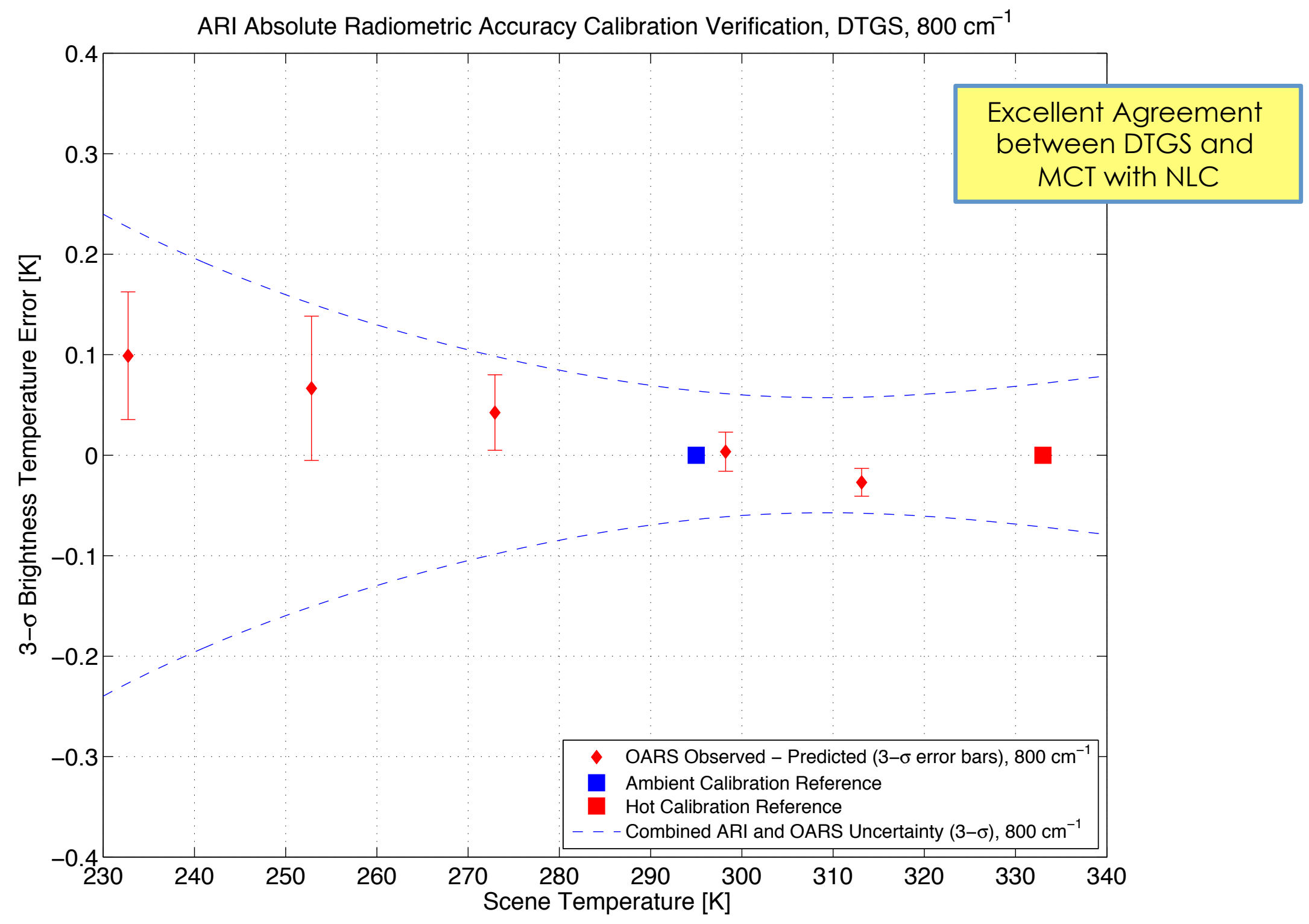




\section{Conclusion}

- An excellent, low cost, climate benchmark mission has been defined

- The proposed IR measurement requirements are supported by good technical readiness

- The UW-SSEC ARI (and OT/V)

- Facilitates the demonstration of the technology necessary to measure IR spectrally resolved radiances $(5-50 \mu \mathrm{m})$ with ultra high accuracy $(<0.1 \mathrm{~K}, \mathrm{k}=3$, brightness temperature at scene temperature) for a benchmark climate mission.

- Subsystems have been selected and developed to provide a system with a clear path to space.

- Initial end to end system tests have been completed - meets radiometric uncertainty goals

- Vacuum testing preparation underway

- This technology can form the basis of a future climate benchmark mission, such as CLARREO or Zeus. 


\section{THANK YOU}

